\title{
EL PAPEL DE LOS GEOINDICADORES EN EL ANÁLISIS ESPACIAL. UNA HERRAMIENTA DE APOYO A LA CIENCIA GEOGRÁFICA.
}

\section{THE PAPER OF THE GEOINDICADORES IN THE SPACE ANALYSIS. A TOOL OF SUPPORT TO THE GEOGRAPHICAL SCIENCE.}

\author{
Prof. Mg. Alberto, Jorge Alfredo \\ Docente Investigador CGA -IGUNNE
}

\begin{abstract}
Resumen:
Los Geoindicadores han sido propuestos a partir de aproximaciones y técnicas estándar usadas en Geología, Geoquímica, Geomorfología, Geofísica, Hidrología, Geografía y otras ciencias abocadas al estudio del planeta Tierra. Los mismos están orientados al análisis de los componentes abióticos de ecosistemas y geoformas, sin embargo, muchos indicadores están íntimamente ligados a ciertos sistemas biológicos, tanto en tiempo como en espacio, a lo que se suma el análisis de la dinámica del sistema antrópico.
\end{abstract}

La investigación de los Geoindicadores y sus variaciones puede ser un medio adecuado para reunir información sobre los cambios pasados y presentes, divulgar y utilizar métodos simples de evaluación y control de las modificaciones espaciales y en consecuencia ambientales, y también ayudar a distinguir entre las variaciones naturales y las inducidas por el hombre.

Palabras claves: Geoindicadores; Herramientas de apoyo; Análisis geográfico; Dinámica del espacio; Alcances

\begin{abstract}
:
The Geoindicators has been developed with it bases in approaches and standard techniques used in Geology, Geochemistry, Geomorphology, Geophysics, Hydrology, Geography and other sciences heading to the study of the planet Earth. The same ones are oriented to the analysis of the physical component of ecosystems and form Earth, however, many indicators are intimately bound to certain biological systems, as much in time as in space, to what sinks the analysis of the dynamics of the system atrophic in the space.

The investigation of the Geoindicators and their variations can be a half appropriate one to gather information about the last and present changes, to disclose and to use simple methods of evaluation and control of the space modifications and in environmental consequence, and also to help to distinguish between the natural variations and the induced ones for the man.
\end{abstract}

Keywords: Geoindicators; Support Tools; Geographical Analysis; Dynamics of the Space; Reach

\section{Introducción}

El concepto de Geoindicadores inicialmente fue brindado por Berger (Berger\&lams, 1996) quién al examinar el contenido de los informes ambientales (State of the Environment) utilizados en Canadá notó el predominio de información hacia los aspectos biológicos y químicos, estos últimos relacionados principalmente con la contaminación resultantes de la acción del hombre a través de su accionar sobre el medio. En relación a lo expuesto, Foschiatti (2010) insiste que la multiplicidad de trabajos que focalizan el análisis de la vulnerabilidad de los grupos humanos y de los elementos que organizan el espacio 
presentan como limitantes, en su mayoría, pasar por alto el papel del medio natural como condicionante de las actividades que se desarrollan sobre el mismo, situación que da cuenta no solamente de la profundidad de esos problemas sino también de las dificultades que existen para abordarlos en los distintos escenarios.

En cualquier lugar del planeta los principales problemas ambientales hacen alusión a la erosión hídrica y eólica, la degradación de aguas superficiales y subterráneas, así como los riesgos naturales relacionados con inundaciones y sequías. Como consecuencia, la calidad de vida se ve afectada en distintos aspectos como salud, educación, vivienda y trabajo entre otros. Estos problemas se acentúan en las áreas urbanas por un aumento en la población, lo que da lugar a una urbanización desordenada y en consecuencia, limitada en servicios; y en la zona rural por constituir un agroecosistema con alta presión de uso, mediana a fuertemente subsidiado. En ambos casos estos problemas se agravan por ausencia o incumplimiento del marco normativo regulatorio.

Como se observa, se vive un período de cambios demográficos y ecológicos nunca vistos en la historia del planeta, existiendo una necesidad urgente de mejorar el conocimiento de tales cambios en los que estamos involucrados. Los Geoindicadores en general, y los Indicadores Ambientales en particular pueden proveer una herramienta esencial para advertir sobre inminentes umbrales que requieren modificaciones de políticas, de las instituciones y del comportamiento humano.

En este marco, el objetivo principal del escrito es caracterizar el papel de los Geoindicadores como herramientas para el análisis geográfico con especial énfasis en el estudio del uso del suelo, sumado a las relaciones que existen entre los procesos de presión de las acciones humanas sobre el espacio. Para ello se lleva adelante una revisión y análisis de material referido a diferentes aspectos geográficos como: el análisis de la dinámica espacial, su organización y marco de estudio, alcances y proyecciones de la aplicación de los Geoindicadores, entre otros aspectos

\section{Los geoindicadores y la importancia de su aplicación en el estudio del espacio}

La ausencia de datos sobre los procesos geológicos y geomorfológicos, entre otros, y el carácter aparentemente aleatorio de las intervenciones antrópicas limitan la elaboración de propuestas de diagnósticos adecuados para el uso, la recuperación, la conservación y la preservación de los recursos naturales.

Los cambios ambientales naturales de los paisajes son resultado de ajustes internos en los parámetros geológicos y climáticos que, a su vez, producen modificaciones en la vegetación y en los procesos y materiales de la superficie terrestre a través del tiempo. Esas interacciones y sus variaciones temporales, espaciales y de magnitud son poco conocidas, a las que se suman las actividades antrópicas que actúan como presiones externas modificando los umbrales de estabilidad del sistema (Coltrinari, L. 2001).

La investigación de los geoindicadores y sus variaciones puede ser un medio adecuado para reunir informaciones sobre los cambios pasados y presentes, divulgar y utilizar métodos simples de evaluación y control de las modificaciones espaciales y en consecuencia ambientales, y también ayudar a distinguir entre las variaciones naturales y las inducidas por el hombre.

En un sentido estricto un geoindicador puede concebirse como una medida física, química, biológica o socioeconómica que representan los elementos claves de un paisaje. Un geoindicador se inserta dentro de un marco interpretativo bien desarrollado y su significado se extiende más allá de la medida que representa, es decir que su importancia dependerá de su relevancia en los procesos de toma de decisiones.

La definición expuesta con anterioridad permite deducir que los geoindicadores representan una innovación fundamental en la mayoría de las ciencias referidas al estudio de la Tierra, debido a la necesidad, a partir de un determinado un nivel base inicial, de seguir 
efectuando medidas que faciliten identificar la evolución en el tiempo y en el espacio de las características escogidas. Cave agregar que el monitoreo de la dinámica del ambiente como sistema complejo, con o sin el uso de geoindicadores, no es una tarea sencilla (Cooke \& DoOrnkamp, 1990), ya que:

- los sistemas naturales son complejos, y por lo tanto dificulta la compresión de los cambios que sufren.

- cada paisaje es único

- la repuesta al cambio de un sistema natural es variable

- procesos y causas diferentes pueden producir efectos y formas similares

- los monitoreos suelen ser muy poco frecuentes o demasiado dispersos.

- los datos existentes suelen ser insuficientes

Teniendo en cuenta los conceptos desarrollados hasta el momento y las problemáticas que estos presentan en su estudio, Berger (1996) para definir y describir un geoindicador propone que la información mínima que debe reunirse sea la siguiente:

1. Nombre del indicador.

2. Descripción (está relacionado con fenómenos o procesos geológicos).

3. Significancia (justificación de su monitoreo).

4. Causa (natural o humana).

5. Entorno natural (zona de vida, formación vegetal).

6. Tipos de sitio de monitoreo (localización recomendada).

7. Escala espacial (área destinada al monitoreo).

8. Métodos de medición (técnicas de campo y de laboratorio).

9. Frecuencia de las medidas.

10. Limitación de datos y de monitoreo (dificultades existentes).

11. Aplicación al pasado y al futuro (potencial para predicción y posible aplicación en estudios paleoambientales).

12. Posibles umbrales (valores en los que pueden ocurrir cambios significativos).

13. Fuentes de información (publicaciones, bases de datos, manuales).

14. Aspectos geológicos y ambientales relacionados (comparaciones con otros ambientes).

15. Evaluación global (posible contribución al monitoreo global del medio ambiente y del desarrollo sostenible).

\section{El papel de los geoindicadores en el análisis geográfico del espacio}

Desde la concepción geográfica y sistémica, los geoindicadores deben proporcionar herramientas que permitan la definición de escenarios que se fundamenten en el conocimiento científico y en la capacidad de análisis y proyección del investigador. En el primero para comprender las raíces históricas, la situación actual y la evolución física-social y en la segunda para proyectar y evaluar los aspectos socio-ambiéntales.

Los geoindicadores son medidas (magnitudes, frecuencias, tasas y/o tendencias) de fenómenos y procesos geográficos que ocurren en la superficie terrestre, y que están sujetos a variaciones lo suficientemente significativas como para comprender los cambios ambientales producidos durante períodos de hasta 100 años, que además pueden ser expresados en escenarios tendenciales y/o deseados en los que se estudian e interrelacionan acontecimientos que nos proporcionan imágenes de las condiciones de vida de la población (GEOIN - IUGS, 2012).

Si bien estos cambios que se producen sobre la Tierra abarcan todas las escalas de tiempo geológico, desde las superiores a los millones de años (procesos naturales) hasta las centenas/decenas de años (procesos antrópicos o antropogénicos), Hamblin (1989) considera 
que los geoindicadores evalúan eventos tanto catastróficos como graduales, siempre y cuando éstos se mantengan dentro del marco del período de una vida humana.

Los geoindicadores han sido desarrollados con base a aproximaciones usadas en Geología, Geoquímica, Geomorfología, Geofísica, Hidrología y otras ciencias abocadas al estudio del planeta Tierra, donde la Geografía se desempeña en relación con las antes mencionadas como la disciplina síntesis del estudio del espacio terrestre.

Los geoindicadores están enfocados al análisis de los componentes abióticos de ecosistemas y geoformas. Sin embargo, muchos indicadores están íntimamente ligados a ciertos sistemas biológicos, tanto en tiempo como en espacio, por lo que algunas veces es imposible diferenciar claramente entre indicadores que describen cambios producidos en sistemas inorgánicos y de aquellos que describen cambios en organismos vivientes. La complejidad de algunos indicadores hace su monitoreo costoso, sin embargo muchos son relativamente sencillos y su monitoreo es posible a bajos costos (GEOIN - IUGS, 2012 y Gupta, A. 2000).

Los geoindicadores pueden ser aplicados tanto en áreas urbanas como rurales para determinar cambios importantes en el paisaje, en forma tal que puedan ser difundidas, en términos sencillos entre planificadores y personas que toman decisiones (Gupta, A. 2000). Es decir, pueden ayudar a determinar impactos ambientales, monitorear ecosistemas de forma continua, seleccionar prácticas de manejo integrado de explotaciones económicas y determinar condiciones de base previas a toda explotación minera, construcción de embalses, vías, canales, obras de desviación de cursos de ríos, desarrollo urbano complementado con servicios e infraestructuras derivadas, en síntesis cambios en el uso del suelo (Coltrinari, L. 2001).

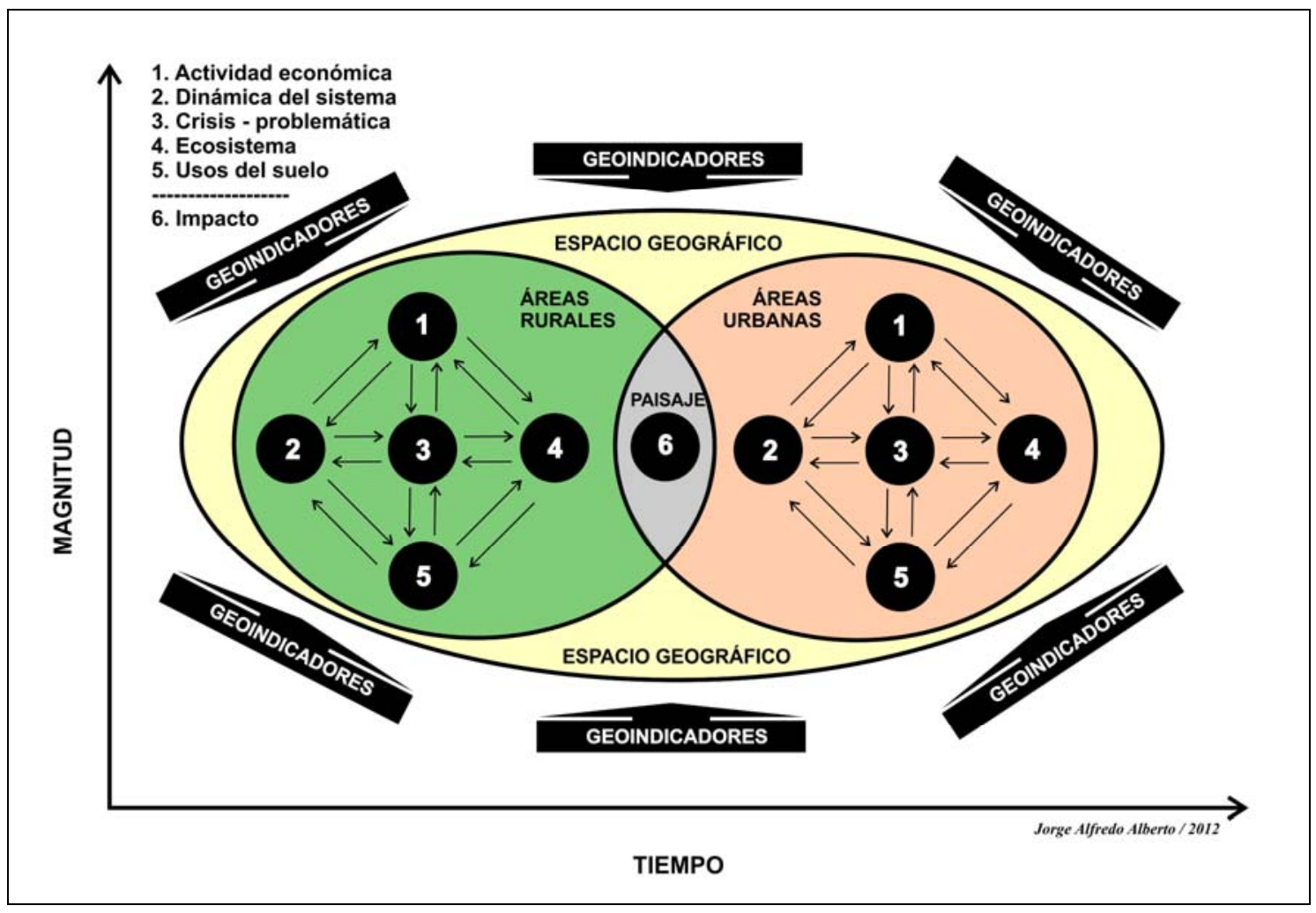

Fig. 1. Aplicación de los geoindicadores en el estudio de la dinámica del espacio geográfico para determinar cambios importantes en el paisaje. Fuente: Berger \& lams, 1996, GEOIN - IUGS, 2012 y Gupta, A. 2000 


\section{Los geoindicadores como herramientas de estudio de la dinámica del espacio.}

Según Gallopín (2006), en la obra "La sostenibilidad ambiental del desarrollo en Argentina: tres futuros" sostiene que "los escenarios (modelos u otros enfoques prospectivos) no representan predicciones del futuro, proyecciones ni pronósticos, sino una exploración de la historia y las trayectorias futuras posibles, con una trama lógica, a partir de la situación actual. Estos serán útiles en la medida que actúen como disparadores de reflexiones sobre los eventos que se plantean en los mismos. Estos se definen también como un curso posible de eventos que lleva a un estado o imagen del futuro". Insiste que "el escenario es una trayectoria mientras que la imagen es una fotografía de la situación futura; el escenario incluye a la imagen y a la historia, como corrientes de sucesos que tratan las alternativas de las acciones humanas y su potencial para afectar significativamente al futuro".

Para el análisis de los escenarios se toma en consideración las evidencias más significativas, ya sean cuantitativas o cualitativas, donde:

- Los geoindicadores permiten evaluar eventos tanto catastróficos como graduales, siempre y cuando éstos se mantengan dentro del marco del período de una vida humana.

- Estos tipos de indicadores facilitan la descripción de procesos naturales capaces de cambiar por sí solos, sin necesidad de intervenciones antrópicas directas, aunque existen formas de acciones humanas que pueden acelerar, retardar o desviar dichos cambios.

- Los geoindicadores están enfocados al análisis de los componentes de los sistemas naturales y antrópicos. Sin embargo, muchos de ellos están íntimamente ligados a ciertos sistemas naturales, tanto en tiempo como en espacio, dificultando la diferenciación clara entre indicadores que describen cambios producidos en sistemas antrópicos y de aquellos que describen cambios en sistemas naturales.

Teniendo en cuenta las aseveraciones antedichas y para analizar las situaciones problemáticas en los diversos escenarios que se desarrollan en el espacio geográfico actual, diferentes autores (Larson, W.E. \& Pierce F.J., 1996; Doran, J.W. \& Parkino, T.B., 1994, Berger, A. 1994, entre otros) proponen abordar las siguientes dimensiones críticas:

- Dinámica del Sistema Natural: El sistema natural es modificada constantemente por el hombre provocando procesos y fenómenos que alteran el equilibrio del ecosistema. Algunos ejemplos notables que provocan la vulnerabilidad del espacio son la saturación de cuencas por deshechos, la contaminación de las aguas subterráneas y de los cursos de agua, la proliferación de cinturones de pobreza en espacios verdes que sirven de pulmón a las ciudades. Ellos motivan la configuración de nuevos paisajes urbanos y rurales bajo riesgo ambiental.

- Dinámica del Sistema Urbano: El análisis del crecimiento urbano y su expansión permite explicar su impacto sobre el medio y las alteraciones del paisaje natural original en detrimento de su calidad y receptividad, como así también su vulnerabilidad física, ecológica y social. Así, algunos de los temas que se tratará para explicar el origen de los problemas ambientales resultantes de la degradación de las cualidades paisajísticas son la degradación de los ecosistemas naturales que alteran la configuración y dinámica del espacio geográfico, la proliferación e incorrecta ubicación de urbanizaciones, la colmatación de cuencas entre otros.

En este marco de hechos, la Geografía desempeña un papel central y las escalas son decisivas para entender la distribución, el impacto y la reducción de los fenómenos a estudiar (Foschiatti, A.M. 2010). Las escalas constituyen un parámetro importante en la detección y seguimiento del impacto y las consecuencias de la misma. La Geografía también explica los vínculos entre los procesos físicos y el contexto humano, y ayuda a definir el alcance del área 0 ámbito espacial. (Beroer, A. R. \& Lam, W. J., 1996) 


\section{Los geoindicadores y su aplicación en el análisis espacial}

La aplicación de geoindicadores en un estudio de enfoque geográfico y sistémico facilita la caracterización de la dinámica de hechos, ya sean naturales o antrópicos, y de las problemáticas resultantes de los mismos observadas en el análisis espacial.

En este aspecto Goudie (1990) expresa que los geoindicadores describen procesos y fenómenos que suceden en el espacio geográfico que son capaces de cambiar con la interferencia humana, ya que esta puede acelerar, desacelerar o desviar la tendencia natural de los mismos y en consecuencia, sin la interrupción de ésta, alterar los ciclos naturales presentes en la dinámica del espacio.

Los indicadores son medidas que hacen perceptible una tendencia o fenómeno que no es inmediatamente detectable en el espacio. La IUGS (International Union of Geological Sciences) decidió, en 1992, a través de su Comisión de Ciencias Geológicas para el Planeamiento Ambiental (Cogeoenvironment), establecer indicadores (magnitudes, frecuencias, ritmos y tendencias) de procesos y fenómenos que ocurren en la superficie terrestre y están sujetos a cambios ambientales que son significativos para utilizarlos en los informes referidos al uso del suelo y al manejo de los ecosistemas. La aplicación de Geoindicadores permite responder:

- ¿Qué pasa en el ambiente? (condiciones y tendencias),

- ¿Por qué ocurre? (causas, vínculos entre influencias humanas y procesos naturales),

- ¿Por qué es significante? (efectos económicos, ecológicos y sobre la salud), y

- ¿Qué hacemos al respecto? (planeamiento y política ambiental).

Algunos de ellos son de "alto nivel" o "integradores" ya que agrupan medidas relacionadas pero independientes. Otros son indicadores "aproximados", ya que representan innumerables condiciones de las que dependen.

Los indicadores primarios pueden usarse solos, permiten detectar y monitorear los problemas más importantes, son representativos del problema que se analiza y en general pueden medirse de forma sencilla y con tecnología de bajo costo.

Los indicadores secundarios corroboran el problema detectado por el uso de un indicador primario, es decir, se sigue el concepto de presión - estado - respuesta en la toma de decisiones. Así, según Berger (1996) los indicadores se desarrollan para evaluar:

- presiones sobre el ambiente debidas a la actividad humana,

- condiciones o estado resultante en el ambiente evaluado, y

- respuestas políticas para corregir situaciones indeseables.

\section{Los geoindicadores: organización de la estructura de interpretación y análisis espacial}

La presentación de un número de geoindicadores requiere de una estructura organizacional que facilite su interpretación y análisis. Ésta organización analítica se desprende de la función de medio de información de los indicadores y depende de la utilidad que deben prestar. Para el análisis de los escenarios se toman en consideración evidencias cuantitativas (datos, modelos matemáticos) y cualitativas (información documental, cartografía de síntesis y análisis), donde intervienen diversos marcos de análisis para indicadores, entre los que se destacan:

- Marco temático (situación actual): consiste en la identificación de hechos o fenómenos específicos para ser analizados (por ejemplo problemas ambientales), es decir, la identificación del tema central.

\footnotetext{
Publicado en formato digital: Prof. Mgter. Jorge Alfredo Alberto. EL PAPEL DE LOS GEOINDICADORES EN EL ANÁLISIS ESPACIAL. UNA HERRAMIENTA DE APOYO A LA CIENCIA GEOGRÁFICA. Revista Geográfica Digital. IGUNNE. Facultad de Humanidades. UNNE. Año 9. No 17. Enero - Junio 2012. ISSN 1668- 5180 Resistencia, Chaco. En: http://hum.unne.edu.ar/revistas/geoweb/default.htm
} 


\begin{tabular}{|l|l|}
\hline Cevista Geográfica Digital. IGUNNE. Facultad de Humanidades. UNNE. Año 9. № 17. Enero - Junio \\
2012. ISSN 1668-5180 Resistencia, Chaco
\end{tabular}

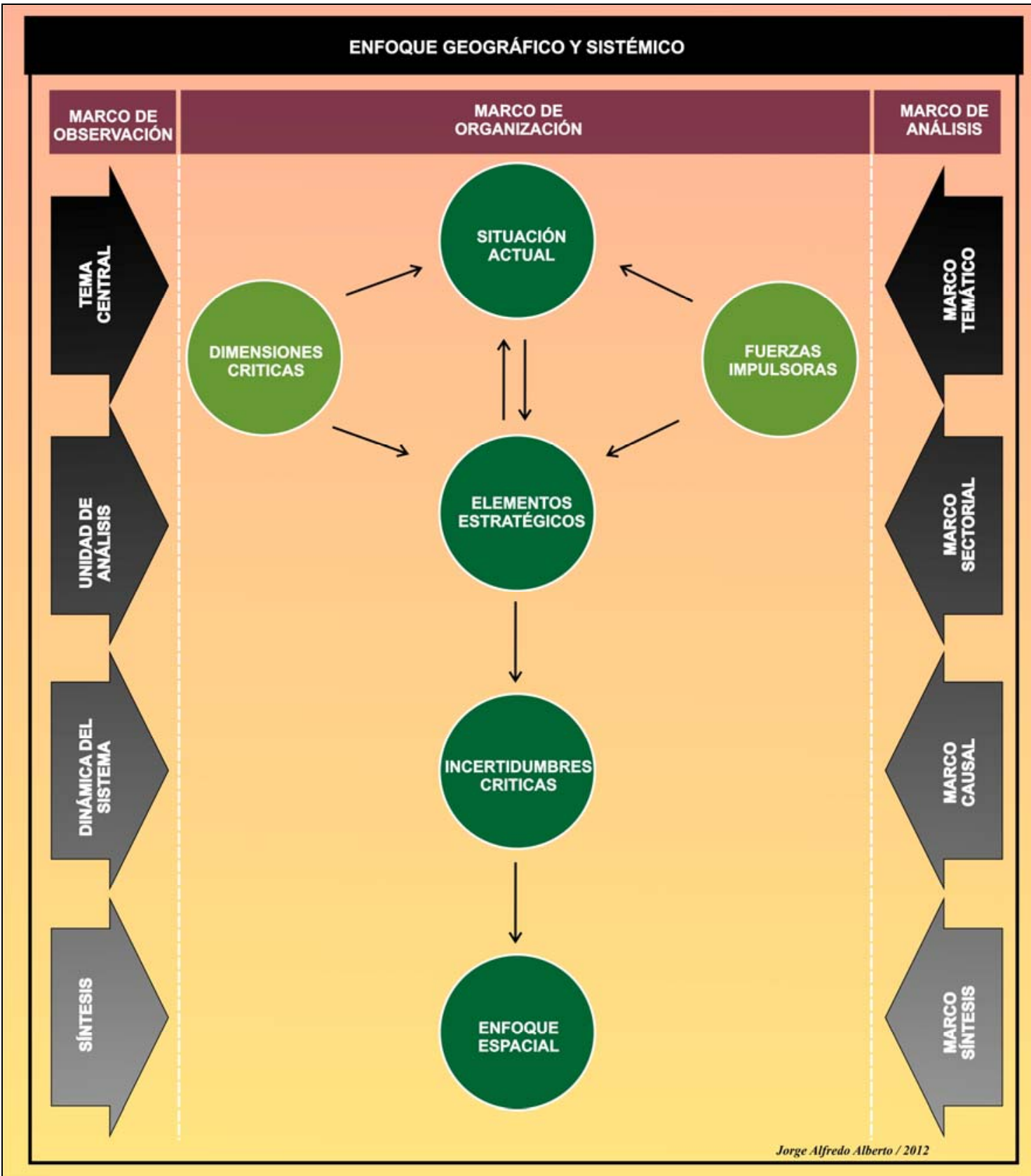

Fig. 2: Desde una concepción geográfica y sistémica para el estudio del espacio, los marcos de análisis facilitan la organización de los geoindicadores. (Fuente: Parada, S. 2005; Berger, A \& lams, W.J. eds,1996; Gupta, A., 2000)

- Estructura por medios (dimensiones críticas y fuerzas impulsoras): criterios de relevancia, descriptores de atributos del medio (recursos como aire, agua, suelo y recursos biológicos). Los indicadores cubren cada medio separadamente como factores, tendencias y procesos claves que influyen sobre los procesos.

- Marco sectorial (elementos estratégicos): se considera al medio como fuente de recursos con una dinámica de los procesos a corto y largo plazo, adjudicándolos a sectores socioeconómicos. 
- Marco causal (incertidumbres críticas): supone que las actividades humanas son fuerzas impulsoras que afectan a los sucesos y por lo tanto ejercen una presión sobre el medio, que se registran mediante cambios de su estado en función de ellas. Para cada una de las acciones se desarrollan indicadores de presión, estado y respuesta.

- Enfoque espacial (argumento y escenarios de futuro): los problemas se clasifican de acuerdo a una vinculación lógica de los elementos que permiten identificar la trama y la dinámica de la situación facilitando el estudio de su incidencia espacial (puntual, local, regional, nacional y global) a partir de la combinación de elementos que ilustran el sistema y su situación futura.

- Marco Síntesis: En la síntesis se realiza la recomposición de todos los elementos del análisis, en sus mutuas correlaciones e interacciones sobre el espacio.

Los marcos de análisis mencionados constituyen diversas formas de organizaciones posibles de indicadores, cuya utilidad depende del uso que se le pretenda dar a la información. Cada indicador posee un efecto sinérgico respecto a la información individual contenida en cada uno, por otra parte hay que señalar que no son excluyentes entre sí.

El marco de análisis dominante es el marco causal, éste se basa en el principio de causalidad (marco Presión - Estado - Respuesta), es decir que las actividades humanas ejercen presión sobre el ambiente y cambian las características cualitativas y cuantitativas de los recursos, que desde la perspectiva geográfica, un elemento importante, imprescindible y lógico es que estos datos sean cartografiados (Parada, S. 2005).

Finalmente el marco de síntesis, es la etapa más interesante del estudio, porque va impreso no sólo el rasgo distintivo de la Geografía, sino también la personalidad del geógrafo. Para llegar a ello será necesario abandonar el clásico esquema lineal, rígido y artificioso, para acentuar aquellos elementos y factores que caracterizan a la realidad geográfica del medio. El mismo, permite señalar como conclusión del trabajo aquellas ideas que puedan ser consideradas válidas en el diseño de propuestas. (Foschiatti, A.M. 2009)

Las ideas o juicios que se expongan habrán surgido de un profundo análisis socioespacial llevado adelante con herramientas que en la actualidad tienen que complementar los estudios de diagnóstico que un territorio pretende tener como meta final: el mejoramiento del bienestar social y la calidad de vida de la población (Foschiatti, A.M. 2010).

\section{Los geoindicadores: organización de los indicadores de Presión, Estado y Respuesta}

En relación a lo desarrollado hasta el momento, se observa, que los geoindicadores pueden soportar efectivamente la toma de decisiones y formulación de propuestas a través de cada etapa del ciclo de toma de decisiones, simplificando datos técnicos, condiciones de comunicación y tendencias, además de suministrar herramientas para una mejor medición de los procesos y/o hechos geográficos.

En términos generales estos pasos forman parte de un ciclo que incluye la percepción del problema, formulación de políticas, seguimiento y evaluación de las mismas, sin embargo, mientras el marco P - E - R (Presión - Estado - Respuesta) tiene la ventaja de eslabonar las actividades humanas, el estado del medio y las acciones encaminadas a la solución de problemas ambientales, tiende a sugerir relaciones lineales entre la actividad antrópica y la interacción con el medio. Esto podría obstruir la visión de relaciones más complejas de los ecosistemas y de la interacción del medio y la economía. De igual manera, un marco puede cambiar a través del tiempo, como un entendimiento científico del incremento de los posibles problemas y como el valor social que envuelve (Beroer \& lams, 1996).

Desde ésta perspectiva, en donde la actividad antrópica se comporta como un factor geológico sobre el medio y el ambiente, se considera apropiado desarrollar tres tipos de indicadores para abordar cada uno de los temas relevantes: 
- Indicadores de Presión: Este tipo de indicadores describe las presiones ejercidas sobre el ambiente por las actividades humanas, como es el caso de las emisiones de gases a la atmósfera y su evolución en el tiempo.

Estos indicadores se clasifican en dos grupos: de presión directa y de presión indirecta sobre el ambiente. El primer grupo corresponde a las externalidades creadas por las actividades humanas, como por ejemplo el volumen de residuos generados y la emisión de contaminantes atmosféricos. Al segundo grupo corresponden tendencias en las actividades que crean externalidades ambientales, como por ejemplo las características de la planta vehicular e industrial. Estos indicadores de presión indirecta también son de importancia pues proporcionan elementos para pronosticar la evolución de la problemática (Beroer \& lams, 1996).

- Indicadores de Estado: Se refiere a la calidad del ambiente, así como a la cantidad y estado de los recursos naturales, por ejemplo, la calidad del aire evaluada a través de la medición de las concentraciones de contaminantes atmosféricos. Este tipo de indicadores incluye los efectos a la salud de la población y a los ecosistemas causados por el deterioro de la calidad ambiental (Parada, S. 2005).

- Indicadores de Respuesta: Presentan los esfuerzos realizados por la sociedad o por las autoridades para reducir o mitigar la degradación del ambiente. Estos indicadores son de los más embrionarios en su desarrollo, debido a la complejidad de medir cuantitativamente como una acción de respuesta contribuye a la solución de un problema ambiental. Las acciones de respuesta son dirigidas hacia dos aspectos, el primero los agentes de "presión", y por otro lado las que se dirigen a las variables de "estado" (Berger \& lams, 1996).

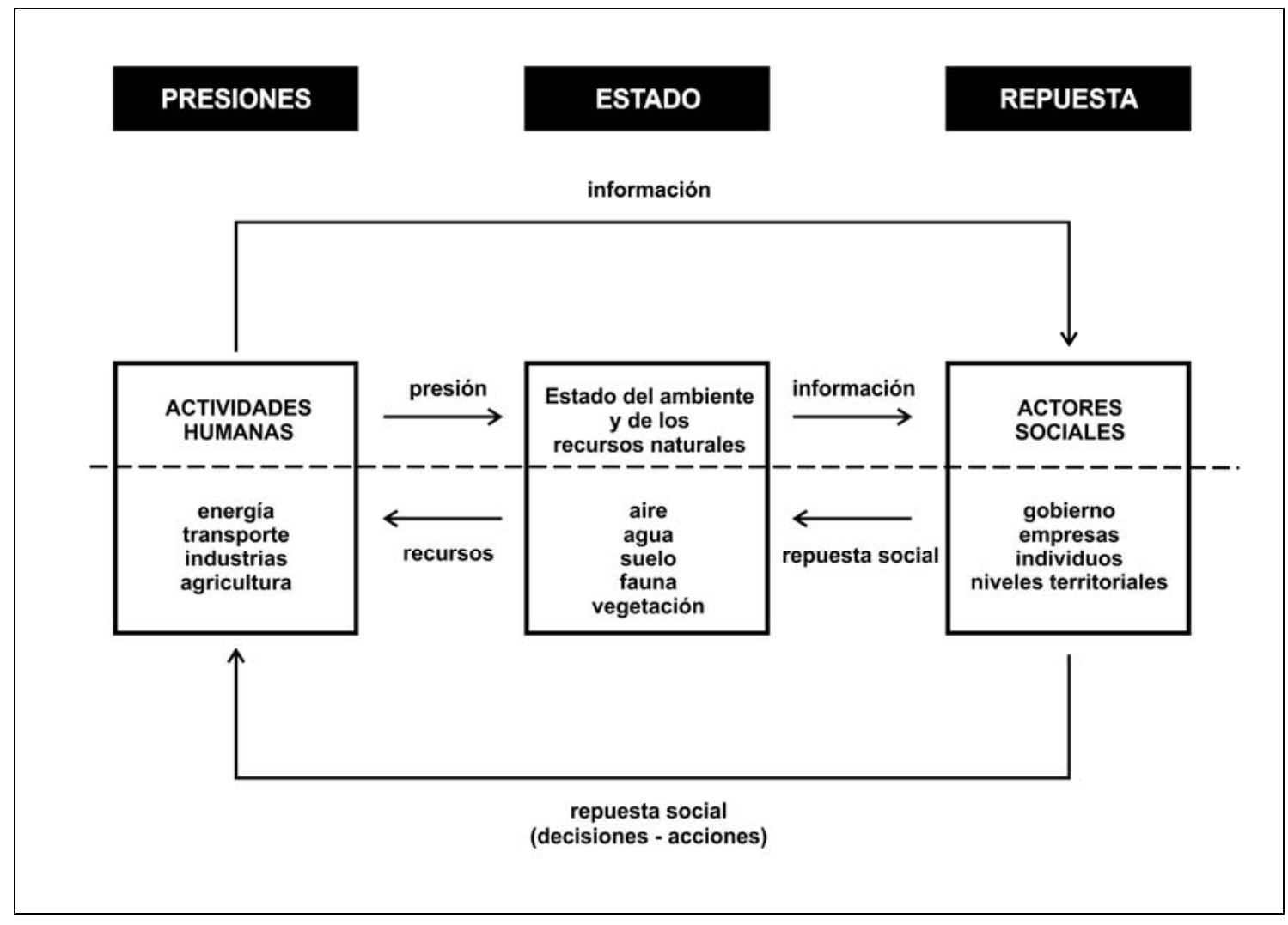

Fig. 3: Organización de los indicadores de Presión, Estado y Respuesta. (Fuente: Gouzee, N.; et al., 1993; Berger, A \& lams, W.J. eds,1996; Gupta, A., 2000) 


\section{Los geoindicadores: organización de los indicadores de Intervención y toma de decisión}

Dentro de los marcos de organización de los indicadores se pueden encontrar tres formas de información para la toma de decisiones, es así como se habla de Indicadores de Logro, Gestión y de Impacto.

- Los Indicadores de logro hacen referencia a las metas obtenidas en relación a las metas planteadas en el período de tiempo antes de la realización de las acciones,

- Los Indicadores de gestión pretenden cuantificar las acciones que en materia de manejo de recursos fueron necesarias para la realización de actividades y alcance de metas,

- Los Indicadores de impacto se refieren al cambio que las acciones emprendidas ocasionaron sobre la realidad social o física.

Los indicadores están sujetos a una serie de criterios derivados de los procesos de toma de decisiones, es decir que dependerán de los objetivos, metas y políticas de orden nacional y regional o local al que pertenezcan. Sin embargo, existen criterios generales para su selección, que se detallan a continuación:

- Reflejar un aspecto fundamental de un hecho o fenómeno del espacio, es decir, brindar una valoración cuali-cuantitativa de un aspecto ambiental.

- Ser aplicable a escala local o regional, sin por ello dejar de brindar su importancia a escala nacional.

- Proporcionar una advertencia sencilla de los problemas potenciales que pueda generar a futuro un hecho natural y/o antrópico sobre la población y el ambiente.

- Ser sensible de monitorearse para proporcionar estadísticas verificables y datos reproducibles que muestren tendencias sobre el tiempo y el espacio.

- Tener validez científica, es decir:

Fácil de entender, aplicar y monitorear.

Ser efectivo en relación a su costo de aplicación.

Ser monitoreado regularmente con relativa facilidad.

Tener relevancia en las decisiones y políticas de manejo.

Contribuir al monitoreo de alcance de metas a corto y largo plazo.

Ser sólido en su aplicación y seguimiento sobre el espacio, flexible en su reformulación y ajustes según necesidades que surjan.

Ser comparable con otras regiones o países de manera anacrónica y diacrónica.

- Finalmente, generar un sistema de geoindicadores para el estudio del ambiente y el manejo adecuado del mismo, para ello deberán proporcionar información acerca de los conflictos de su uso y de las características (físicas, químicas, biológicas y socioeconómicas) de las áreas afectadas por un uso inadecuado y de las áreas con posibilidades de uso.

En relación al último punto, referido a la necesidad de generar un sistema de goeindicadores, se deberá tener en cuenta los siguientes mecanismos de trabajo y aplicación en el siguiente orden:

1. Definir las políticas, planes, programas, proyectos, actividades, objetivos y metas en cuanto a uso del suelo.

2. De no ser así, definir criterios de uso del suelo y para la recuperación de suelos afectados por usos inadecuados.

3. De acuerdo a los dos mecanismos anteriores y considerando los criterios para la definición de indicadores se deberán identificar las áreas en las que el manejo del suelo requiera del empleo de geoindicadores desde la óptica ambiental. 
4. Formular la serie de Indicadores teniendo en cuenta los principios básicos de localización, coordinación y causalidad desde la óptica geográfica de acuerdo al espacio sujeto a estudio e intervención.

5. Realizar la recolección de información necesaria para los indicadores seleccionados teniendo en cuenta aspectos de descripción y caracterización del los hechos o fenómenos sujetos a estudio en el espacio.

6. Generar una base de datos con información sobre las características (parámetros e indicadores) del espacio sujeto a estudio.

\section{Los geoindicadores como herramientas para el estudio del uso del suelo con fines urbanos}

De acuerdo con Gupta (2000) es posible sugerir indicadores para medir los impactos de la urbanización, lo cual no es tarea simple, por las siguientes razones:

1) los geoindicadores fueron creados especialmente para medir cambios geológicos,

2) la evaluación correcta y completa de los cambios debería considerar el ambiente de cada ciudad, y

3) los geoindicadores deben ser utilizados con la intención de llegar al desarrollo sustentable.

Es posible que un cierto número de indicadores destinados a medir cambios asociados con la urbanización (cambios en canales naturales, en el uso de la tierra, o la colecta del excedente pluvial o de agua servida) pueda ser utilizado por todas las ciudades. Sin embargo, es necesaria una lista adicional de geoindicadores para ciudades localizadas en áreas críticas, tales como vertientes escarpadas, valle fluviales de inundación o en las proximidades de bordes de placa activos (Gupta, A., 2000).

Para llevar adelante un estudio sobre el proceso de urbanización de un espacio y su impacto sobre el ambiente los geoindicadores a ser estudiados son:

- El aumento de las áreas con suelo descubierto.

- EL cambio de escurrimiento hídrico fluvial y pluvial.

- La erosión regresiva en las cabeceras.

- El coluvionamiento y aluvionamiento.

- Los cambios en la morfometría y la morfología de las vertientes.

- La velocidad de los movimientos en masa y señales de movimientos futuros.

- Los cambios en los canales (posibles taponamientos, colmatación o erosión lateral).

- El caudal y carga sólida de los ríos.

- Los cambios cualitativos y cuantitativos en la vegetación

- Los índices de destrucción de vegetación (formaciones boscosas, herbáceas, etc.)

- La erosión y sedimentación causadas por procesos naturales o perturbaciones humanas, incluyendo la degradación de espejos de agua naturales (meandros, lagunas, lagos, etc.) por sedimentación y contaminación química.

En este planteo, algunas de las herramientas de trabajo y fuentes de consulta para llevar adelante una investigación de campo son:

- Calicatas y vertientes de cárcavas.

- Instalación de parcelas para estudio de procesos geomorfologicos y registros meteorológicos.

- Descripción y muestreo de suelos y materiales superficiales.

- Mapas y perfiles de vertientes. 
- Levantamiento de transectas de vegetación en campo.

Para completar los datos de campo y/o evaluar cambios en escala local/regional a lo largo de años/décadas, pueden ser adecuadas otras fuentes como:

- Imágenes digitales de satélites para análisis manual o por computadora

- Fotografías aéreas y terrestres

- Interpretación y comparación entre estudios o documentos actuales y pasados

- Comparación entre mapas topográficos, de vegetación, geológicos, geomorfológicos y de suelos, y entre levantamientos actuales y pasados

- Datos geotécnicos, geomorfológicos, meteorológicos e hidrológicos, entre tantos.

- Análisis físicas y químicas, en especial estudios micromorfológicos de suelos y depósitos superficiales

En caso que se deba realizar una evaluación a partir de la prospección de cambios pasados (específicamente desde el Cuaternario Superior - Antropozoica para cuencas sedimentarias de gran magnitud como la chaqueña y la pampeana) para trabajos de tipo geotécnicos complementados con estudios geomorfológicos se deberá tener en cuenta:

- Análisis e interpretación de productos de percepción remota y bases de datos.

- Informes científicos, técnicos, políticos, socio-económicos, que incluyan diagnósticos sobre cuestiones ambientales.

- Disertaciones de cursos de posgrado en geografía, geología, meteorología, ingeniería, geoquímica, edafología, hidrología, arqueología, arquitectura del paisaje, y otros.

- Datos geológicos, arqueológicos, paleontológicos y correlacionados, métodos de datación aplicados a depósitos geológicos.

- Documentos, mapas, fotografías, mensuras, pinturas de paisajes, en archivos, colecciones, museos y bibliotecas oficiales y particulares.

\section{A manera de cierre}

Los paisajes naturales alrededor del mundo han sido transformados profundamente por la urbanización, lo que inevitablemente ha producido efectos en la estructura, función y dinámica de los sistemas ecológicos en una amplia gama de escalas. Es por ello que la acelerada urbanización en el mundo ha vuelto de suma importancia los estudios relacionados con el análisis territorial y las aplicaciones a gran escala (manejo de recursos naturales, planificación del uso de la tierra y conservación de la biodiversidad) que permitan comprender y considerar estas dramáticas transformaciones de la tierra y sus consecuencias ecológicas sobre el paisaje.

Los indicadores son una propuesta relativamente reciente para este tipo de estudio que se ha difundido ampliamente en la última década. En principio se aplicaron para determinar el cumplimiento de acuerdos ambientales internacionales, como los propuestos en la cumbre de Río de Janeiro. Se crearon a partir de la ausencia de métodos evaluativos confiables que determinaran el alcance y nivel de gestión de las políticas, programas y/o proyectos formulados en términos ambientales.

La Geografía, como ciencia síntesis aporta al estudio de los geoindicadores la base para la elaboración, descripción, sistematización e interpretación de los datos cartográficos y estadísticos (demográfico, económico, social, urbano, catastral, fotográfico, documental, etc.) referidos al espacio sujeto a estudio, lo cual permite la representación cartográfica y esquemática de los procesos observados.

Estas expresiones gráficas y cartográficas, a nuestro entender pueden resultar altamente significativas, si se tiene en cuenta que una expresión clara permitirá visualizar con mayor justeza, las variables analizadas dando como resultado la síntesis del trabajo. De 
esta manera se facilita el diagnostico en forma cuali - cuantitativa del fenómeno sujeto a estudio a nivel local y/o regional; ya que se intenta reflejar los resultados de los datos obtenidos y de la información recopilada.

La representación gráfica y cartográfica es un elemento esencial y muy claro, utilizada para explicar numerosos fenómenos y características, especialmente cuando son tratados en su evolución temporal y su distribución espacial. El análisis, la comparación, la superposición, la diferenciación y la síntesis que se pueda lograr en la confección de los mismos, resulta de sumo interés e importancia en la resolución de los temas estudiados por los geoindicadores.

Cabe aquí la observación del Canadá Environmental Advisor Council (CEAC, 1991) en el sentido de que con el fin de incorporar a ellos consideraciones académicas y capacidad predictiva, los geoindicadores deben presentar las siguientes características:

- ser utilizables para prospecciones futuras y no ser simplemente una herramienta de monitoreo

- reflejar enlaces entre el comportamiento humano y el grado de vigor y productividad del sistema natural

- reconocer la incertidumbre inherente al comportamiento de los sistemas naturales y antrópicos.

Varios de esos datos pueden ser difíciles de obtener, sin embargo al plantearse cualquier país, región o gobierno local su intención de buscar un desarrollo sostenible, debe tener claro que esa meta no se logra a menos de disponer de datos confiables sobre la evolución de su espacio geográfico y ambiente, que incluye obviamente los aspectos cubiertos por las geociencias, como ha quedado demostrado en este trabajo.

\section{Bibliografía}

- Bazant, J., (2000). "Periferias urbanas. Procesos de expansión y consolidación urbana incontrolada de bajos ingresos y su impacto sobre el medio ambiente", editorial Trillas, México.

- Berger, A \& lams, W.J. eds (1996). "Geoindicators: Assessing rapid environmental changes in Earth systems." Rotterdam, A. A. Balkema, pp 466.

- Berger, A. (1996). "The geoindicator concept and its application: An introduction". In: Berger, A. \& lams, W.J. eds. Geoindicators: Assessing rapid environmental changes in Earth systems. Rotterdam, A. A. Balkema, p.1-14.

- Bertrand, C., Bertrand, G. (2002). "Une géographie traversière: L'environnement à travers territoires et temporalités", Paris, éditions Arguments.

- Bertrand, G. (1979). "Ecologie de l' espace geographique. Recherches pour une "Ciencie de paysage". C.R. Soc. de Biogéographie. № 406.

- Bolós I Capdevila, M. et Al. (1992). "Manual de Ciencia del Paisaje. Teoría, métodos y aplicaciones". Masson S.A. Barcelona

- Bossel, H. (1999) "Indicators for sustainable development: Theory, Method, Applications". Areport to the Balaton Group. International Institute for Sustainable Development, Winnipeg, Canada.

- Coltrinari, L. (2001). "Geoindicadores de cambios globales rápidos en los trópicos húmedos".en Encuentro de Geógrafos de América Latina, Universidad de Chile, 8., 2001. Santiago (Chile). p. 12. en línea [consulta 09/04/2012].

<http://geodados.pg.utfpr.edu.br/busca/detalhe. php?id=23145>

- Coltrinari, L. (1996). Natural and anthropogenic changes in the Brazilian tropics. In: In: Berger, A \& lams, W.J. eds (1996). "Geoindicators: Assessing rapid environmental changes in Earth systems." Rotterdam, A. A. Balkema, p. 295 - 310.

- CIAT - WORLD BANK - UNEP (1999). "Índice de uso del suelo". 
- Dalal-Clayton, B. y S. Bass (2002). "Sistemas de monitoreo y evaluación. Estrategias de desarrollo sostenible". Nueva York, Instituto Internacional para Medio Ambiente y Desarrollo (IIED)/Organización de Cooperación y Desarrollo Económicos (OCDE)/Programa de las Naciones Unidas para el Desarrollo (PNUD).

- Doran, J.W. \& Parkino, T.B. (1994). "Defming and assessing soil quality". Soil Science Society of America Special Publication No 35.

- Douglas, I. (1985) "Global mega-geomorphology". In: HAYDEN, R.S. ed. "Global mega geomorphology". USA, NASA, CP 2312. p.10-17.

- Foschiatti, A.M.H. (2010). "Las dimensiones de la vulnerabilidad sociodemográfica y sus escenarios". Párrafos Geográficos. Volumen 9. nº 1. En línea [consulta 10/02/2012]

$<$ http://www.igeopat.org/index2.php?option=com_docman\&task=doc_view\&gid=335\&ltemid $=99999999>$

- Foschiatti, Ana M. (2009). "Aportes Conceptuales y empíricos de la vulnerabilidad global en el Nordeste Argentino". Instituto de Geografía, Facultad de Humanidades. UNNE. IGUNNE. Pp. 11 - 41. En línea [consulta 23/03/2011]

$<$ http://hum.unne.edu.ar/publicaciones/instGeo/digitales/vulnerabilidades/index.htm>

- Foschiatti, Ana M. (2004). "Vulnerabilidad global y pobreza. Consideraciones conceptuales". En Geografica Digital Año 1- № 2 Julio - Diciembre 2004. En línea [consulta 23/03/2011] <http://hum.unne.edu.ar/revistas/geoweb/homeig0.htm>

- Gallopín, G.C. (2006). "Los indicadores de desarrollo sostenible: Aspectos conceptuales y metodológicos". Ponencia realizada para el Seminario de Expertos sobre Indicadores de Sostenibilidad en la Formulación y Seguimiento de Políticas. Fodepal, Fao. Santiago de Chile.

- Gallopín, G.C. (2006). "Sostenibilidad y desarrollo sostenible: un enfoque sistémico". Serie medio ambiente y desarrollo $N^{\circ}$ 64, División de Desarrollo Sostenible y Asentamientos Humanos, CEPAL, Santiago, Chile.

- Gouzee, N.; et al. (1993). "Indicators of Sustainable Develop ment for Decision Making". pp. 5 - 8.

- Gupta, A. (2000). "Geoindicators for tropical urbanization". Conferencia realizada en el Simposio "'Geoindicators: Applications to the Humid Tropics'", 31st International Geological Congress. Rio de Janeiro, Agosto 6 - 17, 2000. [manuscrito] 13p.

- Gupta, A. (1993). "The changing geomorphology of the humid tropics". In: Vitek, J.D.; Giardino, J.R. eds. "Geomorphology: The research frontier and beyond". Amsterdam, Elsevier. p. $165-186$.

- Hamblin, A. (1998). Environmental Indicators. p. 9

- Iracheta, A., (1997). "Planeación y desarrollo. Una visión del futuro", Universidad Autónoma del Estado de México, México.

- IUGS (2012). "IUGS Geoindicators Initiative (GEOIN)" en línea [consulta 20/01/2012]. <http://www.Igt.It/geoin/> <http://www.Igt.It/geoin/files/spanish_flyer.pdf>

- Lefebvre, H. (1971). "De lo Rural a lo Urbano". Península. Barcelona.

- Martínez-Salcedo, F. (1995). "Guías metodológicas para la elaboración de estudios de impacto ambiental". Serie monográfica. Centro de publicaciones, Secretaría General Técnica, Ministerio de obras públicas, transporte y medio ambiente. Madrid (España).

- Molion, L.C.B. (1991) "The Amazonia and the global climate". Boletim IG-USP, Pub.Espec. n.8, p. $15-23 . "$

- NASA (1988). "Earth System Science - A closer view". Washington, D.C., National Aeronautics and Space Administration Advisory Council, Earth System Science Committee. 
- Parada, S. (2005). "El sistema de indicadores socioeconómicos de la desertificación. En Pobreza, desertificación y degradación de los recursos naturales". Comisión Económica para América Latina y el Caribe (CEPAL). Santiago de Chile, diciembre del 2005. pp. 139 150. En línea [consulta 23/03/2011] <http://www.eclac.cl/publicaciones/xml/8/24268/lcg2277e_Cap_V.pdf>

- Reynolds, R. (1985). "Tropical meteorology". Progress in Physical Geography, v.9, p. 157186.

- Rigby D, Howlett D. y Woodhouse (2000). "A review of indicators of agricultural and rural livelihood sustainability" [en línea], Department for International Development Research. En línea [consulta 03/05/2011] <http://les.man.ac.uk/ses/research/CAFRE/indicators/wp1.pdf>.

- Smeets, E. y R. Wetterings (1999). "Environmental indicators: typology and overview", Technical Report, № 25, Copenhague, European Environment Agency (EEA).

- Turner II, B.L.; Meyer, W. (1988) "Land, Water, and Society". Earthquest, Summer 1992, Science Capsule

- Vanclay, J.K.(1993) "Saving the tropical forests: needs and prognosis". Ambio, v.22, n.4, p. 225-231. 